\title{
Quand l'enfant se désigne comme sujet
}

\author{
Aliyah Morgenstern \\ Ecole Normale Supérieure Lettres et Sciences Humaines
}

Il s'agit ici d'aborder la grammaticalisation du «parler de soi comme sujet» chez l'enfant, jusqu'à l'utilisation unique du pronom je. Nous travaillerons sur des faits de langues recueillis dans des corpus longitudinaux d'enfants francophones et anglophones enregistrés en milieu naturel, c'est à dire dans leur milieu familial.

Quand on utilise le terme de «sujet», cela permet de référer à quatre notions distinctes : le sujet parlant, le sujet syntaxique, c'est à dire le premier argument d'un verbe (Co), le sujet sémantique (agent, expérient...), et le topique ou thème. Nous sommes à quatre niveaux de la description linguistique. Nous avons donc parfois du mal à nous y retrouver et cela d'autant plus quand il y a superposition ou conjonction du sujet sémantique, syntaxique, sujet parlant et thème de l'énoncé, c'est à dire quand l'énonciateur se désigne lui-même comme sujet. C'est sans doute en partie cette coïncidence possible entre les quatre niveaux qui a donné lieu à toute une tradition linguistique occidentale selon laquelle le langage est produit par un énonciateur qui, quand il réfère à lui-même en tant que sujet, à tous ces niveaux, en français dit je.

Ce je paraît donc extrêmement complexe au niveau théorique. On peut alors se demander comment un petit enfant arrive à conjoindre ces différentes dimensions dans une seule forme et s'auto-désigner avec je. Et pourtant, l'enfant français tout venant, vers l'âge de trois ans dit je, sans même que les parents puissent en parler comme d'un exploit. Il serait étonnant de rencontrer un parent qui raconte: "ah oui je me souviens, c'est le jour où mon fils a dit je pour la première fois". Le premier "maman», l'apparition de son prénom, éventuellement d'un conditionnel ou d'un subjonctif, constitue un évènement, une "catastrophe" dans le sens de Thom, mais pas je. Et pourtant, certains enfants présentant des troubles du langage, certains enfants psychotiques ont souvent la caractéristique de ne pas se désigner à la première personne.

J'ai voulu essayer de comprendre comment se construisait la complémentarité entre ces différents niveaux dans le langage de l'enfant et comment il "parvenait» à s'auto-désigner à la première personne et au "nominatif".

\section{Les différentes auto-désignations}

Mon travail est parti d'un premier constat: entre dix-huit mois et deux ans et demi, des marqueurs variés sont utilisés par l'enfant pour se désigner: absence de forme, voyelles préverbales, prénom, il/elle, moi, tu, je. Entre deux ans et demi et trois ans, l'emploi de je se stabilise et les autres marqueurs tendent à 
disparaître en fonction sujet. Au même moment, les enfants sortent d'un emploi simplement déictique du langage et manipulent différents temps, modalités et aspects. A partir de ces observations, les questions qui se posaient étaient les suivantes:

- Comment rendre compte de la simultanéité des formes durant une période assez déterminée (à peu près de deux ans trois mois à deux ans huit mois)?

- Ont-elles chacune des valeurs différentes au sein des énoncés et dans tous les contextes de production?

Rappelons que l'enfant est confronté à plusieurs problèmes :

a) se désigner, s'identifier autrement que par son prénom

b) marquer qu'il est à la fois sujet de l'énoncé et sujet énonciateur et conjoindre les deux dans une seule forme

c) marquer qu'il est aussi objet de discours, ou thème

d) le marquer au nominatif

Ces problèmes se traduisent par ce que dans la littérature psycholinguistique on appelle les «erreurs» des enfants, les formes «incorrectes», ou ce que je préfère appeler, les formes "non adultes". Les écarts par rapport à la langue adulte vont consister en

- $\varnothing$ non marquage, absence de forme. Une première piste consite à penser que le fait de parler de soi est alors implicite. Il y a un appui sur une consensualité avec l'interlocuteur, "si je ne marque pas la personne, c'est que je parle de moi". Je me suis donc egalement intéressée à la langue des signes dans laquelle on ne marque la première personne que s'il s'agit d'agentivité contrastée car il y a un appui sur le corps du signeur.

- L'utilisation du prénom, qui sauf dans certains cas comme celui de César ou Alain Delon n'est pas la norme en langue adulte pour parler de soi.

- Un autre cas accusatif (moi), génitif en anglais (my)

- Une autre personne, deuxième ou troisième personne.

Afin de conduire des analyses, nous avons effectué un travail d'équipe ${ }^{1}$ sur quatre corpora longitudinaux dont trois en français (Léonard, Guillaume et Juliette) et un en anglais (Peter). Nous avons relevé toutes les formes d'autodésignations dans les corpus d'enfants entre $1 ; 7-1 ; 8$ et 3 ans, puis nous les avons analysées en contexte.

Nous avons montré qu'au cours de son appropriation du pronom de première personne, l'enfant travaille sur deux plans :

- La valeur référentielle (il dit que celui qui est le sujet est lui-même). On trouve des formes très marquées, le prénom et moi, avec une fonction

\footnotetext{
${ }^{1}$ J'ai travaillé avec la collaboration de Mireille Brigaudiot et Catherine Nicolas
} 
contrastive : «c'est moi et pas toi qui fait ». Exemple à 2;02: "Moi fait la photo" (alors qu'on le filme, il veut prendre le camescope et filmer luimême). Cela pourrait être interprété par «c'est moi qui filme et pas toi ». L'auto-désignation a d'abord une valeur argumentale, contrastive. Il y a à la fois consensualité avec l'autre sur l'activité envisagée, sur le prédicat (filmer) et désaccord, discordance sur qui en est le maître (moi et pas toi).

- La valeur énonciative, le marquage du point de vue (l'enfant est celui qui désire, a des projets, pense, prend en charge ce qu'il dit, se positionne par rapport à l'autre).

L'enfant parle de lui en tant que support modal, émettant ses désirs ses sentiments et petit à petit modalise de plus en plus ses énoncés en laissant des traces de son rôle d'énonciateur. Les auto-désignations sont des indicateurs de subjectivité. Pour reprendre une analyse de Danon-Boileau (1994), «l'enfant considère la réalité, ne la juge pas satisfaisante et a recours au discours pour indiquer ce par quoi il souhaite la remplacer.» En début de corpus, des absences de forme et des voyelles préverbales sont principalement attestées dans des énoncés du type: $(2 ; 01)$ " veux zouer à la pâte à modeler ». Puis petit à petit, le pronom de première personne va s'ébaucher sous forme de voyelles préverbales, $[\varnothing]$ puis de $[\mathrm{j} ø],[\mathrm{z} \varnothing]$ puis de $j e:(3 ; 0)$ «j'entends pas maman, tu peux mettre plus fort ? »

Nous avons pu délimiter trois périodes en ce qui concerne les quatre enfants étudiés :

Période I (de 1;7 à 2;3): l'enfant emploie des formes non-adultes.

Période II (de 2;3 à 2;7): l'enfant emploie toutes les formes

Période III (après 2;7) : 1'enfant emploie principalement des formes adultes.

Nous nous intéresserons tout particulièrement à la période II durant laquelle se chevauchent toutes les formes d'auto-désignation. Tous ces écarts vont donner lieu à l'utilisation simultanées de formes différentes durant la même période. Nous pouvons illustrer cette multiplicité d'utilisation par l'exemple suivant:

(1)Guillaume (2;6) est en train de faire un gâteau avec sa mère.

G1: Ze vais tourner la farine. Ça y est, mis partout. T'as tourné! T'as tourné

l'œuf!!

M1: Bien!

G2: $\quad$ Ah ça colle. Ze veux encore tourner un œuf. Encore un.

M2: $\quad$ Tourne amour, tourne.

$G$ regarde $M$.

G3: $\quad$ Ai tourné.

M3: $\quad$ Maintenant il faut encore un œuf.

$M$ est sur le point de casser l'œuf. G tend sa main.

G4: Guillaume i fait casser un œuf!!! 
Guillaume travaille à la fois la valeur énonciative («ze vais tourner la farine » ici l'enfant est support d'un projet qu'il va accomplir) et la valeur référentielle contrastive (Guillaume i fait casser un œuf » que l'on peut gloser par «c'est Guillaume - et pas seulement maman- qui sait casser un œuf et a cassé un œuf »). Mais on peut observer un phénomène particulier, circonscrit à cette période : il s'auto-désigne à la deuxième personne (t'as tourné !), et un peu à la troisième personne. L'enfant emploi donc tu pour référer à lui-même et non à son interlocuteur. La réaction verbale et non-verbale de la mère nous montre qu'elle interprète le $t u$ de l'enfant comme faisant référence à lui-même, et le contexte de l'énoncé rend possible cette interprétation de la forme.

Il est possible de confronter ces hypothèses sur l'acquisition des pronoms au travail de spécialistes du français et de ses différents états historiques, ainsi que de l'oral spontané contemporain. Des comparaisons intéressantes semblent se dégager sur l'emploi d'une valeur "forte" et contrastive des pronoms en moyen français, dans un état de la langue où la présence du pronom sujet n'était pas obligatoire, à une valeur simplement référentielle. Cette évolution semble très similaire à ce que l'on trouve chez l'enfant.

Ce rapprochement entre l'acquisition et les analyses diachroniques rappelle une remarque de Guillaume (1927):

"Ce fait que l'histoire des langues nous refuse, l'enfant l'offre tous les jours à notre observation. Il est vrai que l'imitation d'une langue toute faite est le principe de cette acquisition. Mais cette assimilation ne peut être entièrement passive; elle passe peut-être par certains stades nécessaires dans l'acquisition de tout langage complexe; des mécanismes psychologiques communs jouent probablement dans la vie des langues et dans leur acquisition individuelle, comme celui dont dépendent les formations analogiques."

Nous avons vu comment différentes formes peuvent être employées par l'enfant pour s'auto-désigner au cours de la même séquence. Nous insisterons maintenant en particulier sur les cas où l'enfant utilise l'accusatif ou le génétif à la place du nominatif, ce qui touche à la notion de sujet grammatical, et les cas où il se désigne à la deuxième et à la troisième personne, et où on a donc un travail sur la catégorie de la personne.

\section{Le problème des cas, me et my en anglais}

L'emploi de moi à la place de je est fréquent en français, mais l'anglais se distingue car les enfants emploient soit $m e$, soit $m y$ à la place de $I$.

\section{1) $\mathrm{Me}$}

Nous voyons dans l'exemple suivant comment se fait la transition entre $\varnothing$ et me. La poignée du magnétophone gêne Peter dans son inspection des différents boutons, il la remue dans tous les sens pour l'enlever: 
(2) PAT: you don't like that handle do you?/ Tu n'aimes pas cette poignée n'est-ce pas?

PET: move that thing off move that thing off. / bouge ${ }^{\circledR}$ cette poignée, bouge ${ }^{\circledR}$ cette poignée.

PAT: mmm that handle's in your way./ Cette poignée tegêne.

PET: me move that thing off./ moi enlève ce truc.

Peter dit move that thing off simplement parce qu'il ne veut plus de la poignée du magnétophone car elle le dérange. Son activité qui consiste à tourner la poigné dans tous les sens est accompagnée et dirigée par le langage. Mais comme les paroles n'agissent pas, Peter a besoin de localiser un agent, luimême, ce qu'il fait avec me. Il ne se désigne plus par son prénom comme le font les adultes, mais par me qu'il est le seul à pouvoir utiliser pour se désigner. De plus, dans son premier énoncé Peter ne parle pas vraiment à quelqu'un, il parle pour que ce qu'il dit s'accomplisse (move that thing off). Le dernier énoncé en revanche, s'adresse à PAT qui vient de lui adresser la parole, l'enfant localise donc l'agent de l'énoncé POUR elle.

Or dans les énoncés de l'enfant, me appartient à une catégorie qui a priori n'existe pas dans le langage de l'adulte. Il l'emploie devant un verbe. Nous pouvons alors nous demander pourquoi Peter a recours à $m e$, quelle valeur il lui attribue et quelles sont les opérations effectuées grâce à ce marqueur.

Me est la réponse à une question en who?

(3) PAT: who's gon (t)a go home? Qui va rentrer à la maison?

PET: me go home. / Moi rentre à la maison.

Me sert à opérer un contraste agentif. $(2 ; 3,21)$

(4) PET: me working a railroad. Daddy's working a railroad. / Moi travaille au chemin de fer. Papa travaille au chemin de fer.

Il semblerait que Peter extrapole la valeur de contraste de me en employant le marqueur en fonction d'agent. Si on regarde l'énoncé de façon stricte, on peut par ailleurs constater que l'on a d'un côté me et de l'autre Daddy's, comme si me était l'équivalent de I'm et tenait lieu de marquage de première personne pour le sujet et l'auxiliaire. La structure me + verbe + complément n'existe pas en tant que telle dans le langage adulte. S'agit-il purement d'une création de Peter?

L'exemple suivant peut nous aider à comprendre les origines de la formation de cette structure:

(5) PAT: you want me to make a car? ok. oh you want Lois to have some paper?/ Tu veux que je fasse une voiture? OK. Tu veux que Lois ait du papier?

PET: Lois have some paper. / Lois avoir du papier. 
PAT: there, that's a nice car isn't it?/ Voilà. C'est une belle voiture, non?

PET: me make a car. / Moi fait la voiture.

PAT: ok. you make a car. / OK, c'est toi qui fait la voiture.

Dans you want Lois to have ou do you want me to write, les marqueurs Lois et me font à la fois partie d'une structure objet du verbe to want, et agents à l'intérieur de cette structure des verbes to write et to have. L'enfant ne marque que la valeur d'agent mais garde la forme me qui est peut-être une trace de la structure englobante. L'adulte, PAT, reprend d'ailleurs la même structure que l'enfant : ok, you make a car. Cet énoncé à la forme affirmative avec le you accentué, est correcte en anglais dans ce contexte, mais évidemment pas avec me. Par contre on pourrait trouver:

(6) Me? Make a car! You must be crazy!/ Moi? Faire une voiture? Tu es fou! Et même:

(7) Me? Make a car? Of course I can do it! / Moi? Faire une voiture, Mais bien sûr que j'en suis capable!

Dans ces énoncés, grâce à la juxtaposition de me et de la base verbale l'énonciateur opère une déconstruction et met en question la relation prédicative. Cela crée une indétermination qui permet d'envisager la non validation du lien entre le sujet et le prédicat. Or c'est justement la validation que l'enfant ne peut pas pleinement prendre en charge. Il a besoin du "ok" de l'adulte pour passer à l'acte. Il n'est donc pas si étonnant qu'il emploie justement le marqueur me associé à la base verbale.

La structure me + verbe + complément n'est donc sans doute pas un choix arbitraire de l'enfant. Nous pouvons voir par ailleurs qu'elle est dérivée de structures plus complexes employées par l'adulte. Mais il l'extrapole à un contexte plus large: $(2 ; 3,0)$

(8) PET: want me get it?/ veux que je le prenne?

MOT: ok. / D'accord.

PET: wait me sit down. ok?/ Attends moi s'asseoir.

MOT: do you want me to wait till you sit down?/ Ext-ce que tu veux que j'attende jusqu'à ce que tu sois assis?

PET: right./ C'est ça.

MOT: ok. / D'accord.

Bien que want me get it et wait me sit down aient la même structure $\varnothing+$ verbe + me+ verbe, la forme "correcte" de ces énoncés n'est pas la même (Do you want me to get it? Wait for me to sit down.). Cependant, le me est justifié par sa double fonction d'objet et d'agent.

L'emploi de me constitue une stratégie pratique et riche en possibilités exploitées par l'enfant. 
Peter comme beaucoup d'enfants anglophones, emploie la forme my qui dans le langage adulte est un adjectif possessif signifiant mon/ma, comme désignation sujet. Il y a deux interprétations possibles, qui peuvent être complémentaires, de ce phénomène:

- My est une sorte d'étape dans la construction du langage qui réunirait me et $I$ ou I'm sur le plan sémantique et phonétique. (Cela explique que l'on ne trouve pas les formes "mon" ou "ma" en français dans le même contexte).

- Pendant toute une période, les enfants emploient énormément de possessifs.

Or ils ne font peut-être pas la différence entre la possession d'un objet et celle d'une action et disent aussi bien my toy que my open. C'est le cas de Peter :

(9) PAT: let's see you'd like some paper, huh?/ Voyons, tu voudrais du papier? PET: write. / Ecrire.

PAT: now let's see, maybe if you get a magazine. / Voyons voir, peut-être que si tu vas chercher un magazine.

PET: huh. my get magazine. / Ouais, "mon" chercher magazine.

(il court de l'autre côté de la pièce pour chercher un magazine).

On voit bien ici que l'alternance des rôles n'est pas YOU/I mais YOU/MY.

Peter remarque que Loïs est en train d'écrire.

(10) PET: my writing. / "mon" écrire.

PAT:no Lois [!] is writing. / Non, c'est Lois qui écrit.

PET: my writing. / "mon" écrire.

Dans le corpus, on voit que Peter commence à utiliser la forme en ING et en $\mathrm{BE}+\mathrm{ING}$ avec des procès pour ancrer son activité dans la situation d'énonciation et il fonctionne comme un inaccompli. Mais ici, cette forme nous montre combien les verbes et les noms sont proches, puisque my writing pourrait se traduire par "mon écriture" (comme si l'enfant actualisait le résultat du processus) alors que PAT l'oppose à Lois is writing, où ING fait partie d'une forme verbale. Nous sommes ici sur une sorte de frontière entre le nom et le verbe où l'enfant effectue l'opération de possession avec un effet de sens de contraste. On trouve d'innombrables my do it, my write, my car, my name, my open..

Il semble donc y avoir une opération supplémentaire dans my par rapport à $m e$ car le premier garde sa valeur de possession. Au lieu de posséder un objet comme dans my piece ou my book, Peter possède le processus marqué par le prédicat: my do it, my go home.

Les limites entre la possession "nominale" et cette possession "verbale" ne sont pas très nettes, tout comme les limites entre un item nominal et verbal: $((2 ; 2$, 14)

(11) PAT: it's too cold to go home. / Il fait trop froid pour rentrer à la maison.

PET: my go home. this my go home. this is my home. / mon rentrer à la maison. C'est ça mon rentrer à la maison. C'est ça mon rentre à la maison. 
PAT: yes it is./ Mais oui c'est ça.

\section{3) $I$}

On peut constater une différence entre les marqueurs $I$ et me dans l'exemple suivant.

(12) PAT: ok you found it. / Eh oui tu l'as trouvé.

PET: me found it . I find it. Moi l'ai trouvé. Je le trouve.

Me prend exactement la place de you. Quand l'enfant parle en situation, il utilise le prétérit pour référer à l'acte accompli et localise l'agent avec $M E$. Mais ensuite il fait une sorte de "synthèse" et se donne une qualité: la forme au présent générique I find peut vouloir dire "je suis un trouveur".

Il s'agit ici de donner une interprétation énonciativement riche de l'emploi des différents marqueurs. Je me permets de faire ce genre d'analyse parce que j'ai pu faire un relevé de toutes les formes et constater des "invariants". Mais on peut aussi penser que l'enfant est en phase de "babil d'énoncé" et essaye les différents marqueurs pour ensuite n'en garder qu'un.

Nous avons regardé comment un enfant anglophone utilise des «cas » différents pour s'auto-désigner alors qu'un adulte emploierait le nominatif. Nous allons analyser maintenant les énoncés d'un enfant francophone qui emploie la deuxième ou la troisième personne pour se désigner à la place de la première.

\section{Le problème des personnes : tu et il}

\section{1) Le récit autobiographique}

Certains enfants utilisent les formes il parfois associées au prénom. Ils parlent donc d'eux à la troisième personne, marquant ainsi le délocuté mais ne l'identifiant pas au locuteur, au sujet parlant. Or il s'agit là de $30 \%$ des autodésignations durant la période II chez Léonard, l'un des petits garçons français dont j'ai analysé le corpus, donc c'est un phénomène quantitativement important.

Une explication des différences basée sur le système linguistique luimême ne peut rendre compte de ces différents emplois dans le récit. Il nous faut donc trouver une explication fondée sur la spécificité du genre narratif. On est dans un système que l'on peut appeler monologique dans le sens où l'enfanténonciateur est ou pense être le seul à savoir et fait savoir à l'autre par sa bouche.

En étudiant systématiquement les énoncés à la troisième personne de Léonard ${ }^{2}$, j'ai pu constater que

- $\quad$ l'enfant a recours au $i l$ quand il est dans une situation de récit qui suppose la mise en mot d'une sorte de projection cinématographique des évènements qui

\footnotetext{
${ }^{2}$ Ce corpus a été recueilli dans le cadre de mon doctorat; Morgenstern 1995.
} 
se redéroulent dans sa tête. Les images qu'il revoit provoquent le même sentiment d'altérité que l'image de soi dans le miroir ou sur une photo. Or on sait qu'à la même époque, l'enfant ne se désigne pas en disant je ou moi quand il parle de son reflet dans le miroir ou sur une photo. (René Zazzo).

Voici un exemple de il pour je chez Léonard.

(13) Léonard 2;02.

Léonard est sur le point de prendre le bain. Il est déshabillé, dans les bras de sa mère.Elle l'embrasse puis il frotte son bras comme pour effacer le baiser, puis porte son doigt sur son visage entre les yeux.

L: ladipan à Davib //

M: T'as dit pan à ...

L: ladipan à Dadib //

$P$ entre dans la salle de bain.

$\mathrm{P}:$ T'as dit pan à David?

L: oui //

$\overline{\mathrm{M}}$ : David de la crèche?

$\underline{\mathrm{L}}$ : 1 eona ila / leona adipan à Dadib //

M: Pourquoi?

L: oui //

M: Pourquoi t'as dit pan à David? David il t'avait dit pan aussi?

L: pabo Dadib pabo a Dadib //

Dans la séquence précédente, Léonard a été amené à raconter ce qui est arrivé à la crèche à partir d'une question de ses parents :

P: Qu'est-ce que t'as fait à la crèche aujourd'hui?

L: atata Xx la crèche l'a fait l'a fait / la pâte à modeler //

La question lui montre que ses parents ont besoin d'avoir une information qu'il est seul à connaître, car ils n'étaient pas témoins des événements. Il est donc entré dans le mode narratif. Puis, au moment de se préparer pour le bain, alors qu'il était tout nu dans les bras de sa mère, il a été un peu agressif avec elle, mais sur un mode taquin: elle lui faisait des bisous sur le bras et il jouait à les effacer avec la main en disant "pas bisous sur les bras".

Il va conjoindre le mode narratif et son humeur un peu agressive en réinitiant l'entrée dans le récit à partir d'une association gestuelle et visuelle. Il donne l'impression de revivre la scène d'autant plus qu'il ponctue ses énoncés de gestes violents montrant qu'il a frappé David au visage. Il donne donc à voir la scène à ses parents en la racontant et la mimant et en se représentant comme un personnage au même titre que David. Cette distance entre l'enfant sur le point de prendre son bain à la maison, et l'enfant de la crèche est marquée par l'usage de la troisième personne. Par ailleurs, puisque le petit garçon a été filmé, j'ai pu constater que quand il produit cet énoncé, il ne regarde pas du tout son interlocutrice. Son regard est vague, comme s'il était concentré sur la scène qui se déroule dans sa tête. Cela nous a rappelé ce que dit Cuxac () sur le récit en 
Langue des Signes Française. Quand le signeur est engagé dans une activité de récit, il ne regarde pas son interlocuteur.

Puis on a l'explicitation de l'identité des deux protagonistes d'abord par la question de M "David de la crèche?" Léonard ne répond pas directement mais localise explicitement un autre agent en reprenant l'usage d'un prénom, le sien:

L: leona ila / leona adipan à Dadib //

On voit donc que Léonard emploie son prénom pour parler de l'agent une fois qu'il a besoin de marquer la place des personnages dans la scène. En tant que celui qui raconte, il partage le point de vue de ses parents et porte un regard d'observateur sur la scène, il se nomme donc par son prénom, comme si un autre que lui racontait la scène. Ce dédoublement lui permet de bien distinguer le plan de la journée passée à la crèche et le plan de son présent à la maison. Mais tout en racontant il mime, ce qui crée un lien entre le Léonard "parleur" et le Léonard "acteur". Le corps de Léonard énonciateur (et surtout son bras) sert de support pour montrer à sa mère le Léonard personnage de son récit. Cela rappelle aussi le récit en langue des signes ou le signeur fait ce que Cuxac appelle un transfert personnel et prête son corps au personnage.

La mère a eu un rôle important d'abord parce qu'elle a introduit le plan du récit par sa question « qu'est-ce que t'as fait à la crèche aujourd'hui »; Ensuite parce qu'elle pose des questions qui amène Léonard à poursuivre son récit, à le commenter, à justifier ce qu'il a fait. Toutes les prises de parole de l'adulte sont des questions qui entraînent une continuation et un enrichissement du thème. $\mathrm{Ce}$ qui intéresse la mère, c'est le récit de ce qu'a fait son fils en son absence et ses justifications, ce n'est pas comment il se désigne. Il y a une forte sollicitation de sa part.

Il est intéressant ici de voir que Léonard emploie la troisième personne dans ce que l'on peut appeler "le récit adressé à sa mère". Il utilise la marque qu'elle utiliserait pour raconter elle le récit à un tiers, il adopte son point de vue à elle sur sa personne à lui. Mais il s'agit justement d'un récit qu'elle ne peut pas raconter, puisqu'elle n'en a pas été témoin. Dans ces contextes où il y a peut-être une stratégie d'évitement de la prise en charge, du marquage modal, du modus, puisqu'on est dans des bêtises, la prise en charge est en quelque sorte dans l'information que l'enfant est seul à pouvoir donner, ce dictum qui lui appartient seul. Et c'est ce dictum qui est fortement sollicité par sa mère en tant qu'il est propre à l'enfant. C'est non pas dans le marquage du pronom que l'enfant inscrit son identité, son processus de séparation-différenciation, non pas dans le sujet, le thème de son propos, sur lequel il y a consensus puisque tous deux savent qu'il s'agit ici d'autobiographie, mais dans le rhème, dans cette histoire qu'il raconte lui. Sa mère lui montre par ses questions et ses demandes de justifications qu'elle sait qu'il vit des expériences en solitaire (c'est à dire séparé d'elle), qu'il a des états intérieurs et qu'il peut les formuler. Elle exprime ainsi qu'elle peut se représenter qu'il a des représentations à lui. 


\section{2) Tu pour JE}

On trouve, tout au long de la littérature sur l'acquisition des pronoms personnels un intérêt pour les «erreurs » des enfants. Il s'agit de "profiter des erreurs des enfants" pour se poser les bonnes questions dont les réponses permettraient d'éclairer le mécanisme d'acquisition des pronoms personnels. (Chiat 1989). Quand il n'y a pas d'erreurs, cela montre que ce qui est linguistiquement complexe ne l'est peut-être pas "psychologiquement" ou cognitivement. Par ailleurs les «erreurs", ou plutôt les décalages entre l'incorrect chez l'enfant et le correct chez l'adulte, permettraient de faire des hypothèses sur les concepts que l'enfant se construit à propos des pronoms.

Les renversements pronominaux sont un phénomène marginal (peu d'enfants les font et ils les font peu) mais considéré comme très important. Par ailleurs, c'est un phénomène que l'on rencontre également chez les enfants aveugles et autistes. Une étude chez l'enfant tout venant peut éventuellement faire comprendre d'où viennent les erreurs en pathologie: "the pronoun reversing child, rare as she may be, is surely one of the most valuable sources of information about this area of development" (Chiat 1986).

Ursula Bellugi a été la première à constater ce phénomène chez les enfants sourds signeurs le jour où une mère et sa fille (de moins de 2 ans), toutes deux sourdes, vinrent lui rendre visite dans son laboratoire. La petite fille signa TOI alors qu'elle voulait dire MOI. Sa mère corrigea l'orientation du signe en lui prenant la main et en la tournant de façon à ce qu'elle touche la poitrine de l'enfant. Mais par la suite, la petite fille continua de faire la même "erreur". Laura Pettito $(1984,1986,1987)$ poussa encore plus loin cette observation dans son travail de thèse et dans sa recherche en Langue des Signes américaine et en Langue des Signes québécoise. La nature iconique des signes ne faciliterait pas l'acquisition des pronoms ce qui signifierait que l'enfant ne tient pas compte de l'information physiquement présente dans les pronoms-index. Au contraire, il traiterait le pronom comme un nom d'objet dont la référence est toujours la même et non comme un "shifter". Il s'agit donc d'un problème linguistique. Bien que la relation entre la forme symbolique, le pointage et son sens soit directe et explicite, elle n'est pas évidente pour les enfants qui l'intègrent dans le système linguistique de la Langue des Signes. L'acquisition des pronoms personnels se passerait donc de la même façon en Langue des Signes et en langue orale.

Comme nous l'avons vu dans l'exemple rapporté par Bellugi de la mère qui corrige le signe TOI de sa petite fille alors qu'elle voulait dire MOI, l'enfant sourd rencontre un problème important lors de son acquisition de la Langue des Signes: ses gestes doivent être symétriquement opposés à ceux de son interlocuteur. Quand un adulte signe MONTRER par exemple, la paume de la main dominante est tournée vers l'enfant. Pour répéter ce signe, l'enfant doit tourner sa paume de main vers l'adulte et non vers lui-même. Ceci est à mettre en relation avec les enfants qui pointent l'autre au lieu de se pointer eux-mêmes 
pour dire je car ils ne renversent pas le signe en se l'appropriant de leur point de vue, mais imitent le mouvement et la configuration de la main tels qu'ils les voient. Mais comme en langue orale, ces erreurs ne sont pas systématiques.

Or Chiat (1982) présente une hypothèse qui prend en compte le fait que les erreurs ne sont pas systématiques et fréquentes. Elle pense que les enfants comprennent parfaitement bien la nature déictique des pronoms et que leurs erreurs sont un usage qui traduit un changement de perspective mentale. Chiat analyse les productions d'un petit garçon Matthew et montre qu'un grand nombre de renversements ont lieu quand l'enfant exprime le point de vue de l'autre. Ses $t u$ référant à lui-même se produisent dans des contextes où Matthew exprime ce qu'il entend d'habitude de la part de l'adulte, c'est à dire des contextes de menace, de permission, d'accusation ou de mise en garde.

Nous allons explorer cette hypothèse en analysant plus en détail des extraits du corpus de Guillaume ${ }^{3}$ durant la période II, celle pendant laquelle il utilise plusieurs formes d'auto-désignations.

(14) Guillaume $2 ; 3$

$G$. fait l'inventaire des chaussures et il commente.

$\mathrm{G}$ - C'est à papa.

M - C'est les souliers de papa.

$\mathrm{G}$ - Peux, veux à mettre, veux.

$\mathrm{M}$ - Tu veux les mettre?

$\mathrm{G}$ - Oui met les souliers de son père comme ça marche

$\mathrm{M}$ - Hein?

$\mathrm{G}$ - se met debout avec les souliers Marcher comme ça

$\mathrm{M}$ - Tu veux marcher comme ça?

$\mathrm{G}$ - Ouais marche quelques pas bravo tu marches !!

On remarque que tant que Guillaume présente son désir et son projet, on trouve $\varnothing+$ verbe. Une fois qu'il a commencé à accomplir son acte, on trouve une forme marquée qui est $t u$. Est-ce un $t u$ qui signifie $j e$ ? Est-ce que vraiment c'est à l'enfant de se congratuler? Est-il l'énonciateur approprié, cet énoncé ne revient-il pas à la mère ? On est dans un contexte d'exploit. Cet énoncé ressemble fort à du discours entendu. On peut dire que l'enfant exprime sa propre fierté en empruntant le rôle de congratulateur habituel de sa mère. Le langage lui permet ainsi d'être dans cet espace intermédiaire entre la fusion avec l'autre et la séparation. Cela passe par un énoncé dans lequel il parle de luimême comme si un autre lui adressait la parole. On a donc une forme tu qui marque à la fois l'altérité et l'identité.

La mère continue le dialogue sans poser de question sur la référence de ce TU. Elle l'a bien compris comme si l'enfant avait dit $j e$.

(15) Guillaume $2 ; 5$

\footnotetext{
${ }^{3}$ Ce corpus a été recuilli par Mireille Brigaudiot.
} 
Guillaume est en train de manger une cacahuète.

$\mathrm{G}$ - t'as avalé encore !

$\mathrm{M}$ - non, une seule cacahuète, pas tout.

On est dans un contexte de betise, frôlant le danger. Ce $t u$ se retrouve quand l'enfant court sur le trottoir, s'arrête au moment de s'élancer sur le passage clouté et dit «tu traverses pas». Cela nous rappelle également le «non» ou même le hochement de tête que fait l'enfant autour de 1 an alors qu'il a levé la main pour toucher un radiateur, une prise électrique ou tout objet dangereux. On voit bien ici que l'enfant produit un bout de "script" qui correspond à une situation. Il fonctionne avec sa mémoire auditive associée à la citation, ou plutôt il associe une forme [tu+prédicat] à une situation. C'est comme s'il empruntait la réplique de sa mère et sa place de locutrice. L'important n'est pas qui parle mais que l'énoncé soit produit. A partir du moment où le texte existe, où il n'y a pas à le créer et où il s'applique à la situation en cours, il le dit.

Laurent Danon-Boileau (2002) rapporte l'énoncé d'un enfant qu'il suit en thérapie qui pour conjurer l'étrangeté d'une situation à laquelle il est confronté se sert d'un énoncé qui lui a sans doute été adressé. Sa mère lui tend la salière alors qu'il avait demandé du sucre pour son yaourt et il dit « tu t'es trompé de pied » puisant ainsi «dans une situation antérieure des images et des mots pour circonscrire ce qu'il ressent cette fois quand sa mère lui tend le sel »(p.65). Dans ce cas, le tu sujet de l'énoncé pourrait être considéré comme «correct» puisque l'enfant s'adresse à sa mère, mais l'emploi du prédicat « tu t'es trompé de pied » nous montre que cet énoncé fonctionne de la même façon que ceux que nous décrivons dans le corpsu de Guillaume.

Avec le tu "pour je», nous sommes également dans l'utilisation citationnelle qui s'appuie sur la mémoire auditive d'un script, d'un scénario, il s'agit d'une citation d'un discours adressé à soi-même. L'enfant a intégré un format dans lequel la question de la référence du locuteur est encore seconde pour lui.

Il arrive cependant un moment où se pose dans le dialogue la question de la clarification de la référence. Cela coïncide avec un moment métalinguistique.

(16) Guillaume $2 ; 7$ (dernier « tu pour je » du corpus)

$G$ et $M$ évoquent des jouets non présents

$\mathrm{M}$ - y'en a beaucoup ! i sont de quelle couleur?

$\mathrm{G}$ - bleu

$\mathrm{M}$ - oh c'est pas vrai

G rit - t'es menteur !

$M$ - t'es un menteur, ah ah ah, t'es un menteur ! i sont pas bleus i sont rouges !

$\mathrm{G}$ - i sont rouges, menteur !

$\mathrm{M}$ - petit menteur, tu joues hein ma puce

$G$ - t'es un petit menteur.

$M$ - t'es un petit menteur

$\mathrm{G}$ - maman t'es un menteur. 
M - ah moi j'suis pas menteuse non. (...)

$\mathrm{G}$ - non, papa i dit p'tit menteur papa, après idit p'tit con

Ici il y a encore citation " t'es un petit menteur» mais dans une visée ludique. La parole de l'autre est repensée, manipulée. L'enfant effectue même une opération de comparaison entre la parole de la mère, la parole de l'enfant et celle du père. Cela montre une distance par rapport au discours rapporté et nous fait comprendre pourquoi il s'agit dans le corpus du dernier tu pour je de Guillaume. A travers le discours rapporté, l'enfant peut devenir un porte-parole. La parole de l'autre est extériorisée, reformulée et non plus imitée ou imaginée. Ce corpus se termine avec des jeux entre mère et enfant sur la clarification de la référence. Il y a donc un moment où la distance est établie et le jeu métalinguistique est possible.

A un certain moment de la genèse, chez l'enfant tout venant, ce phénomène de renversement pronominal prend fin. Il ne se désigne plus que par le pronom de première personne, conjoignant ainsi son rôle de sujet-énonciateur, de sujet de l'énoncé et d'objet de discours dans une seule forme. Pour comprendre cet événement, il faut croiser différents paramètres et observations.

Dans le dialogue avec la mère, on peut voir le marquage d'un travail à deux sur la clarification de la référence du sujet syntaxique.

Par ailleurs, comme nous l'avons montré, les emplois de $t u$ sont liés à la mémoire auditive et ils s'inscrivent dans des scripts avec une succession de répliques. C'est le souvenir d'une situation qui déclenche l'énoncé. L'enfant abandonne le $t u$ ainsi que le il/elle pour s'auto-désigner à partir du moment où il peut se détacher de la situation figée et concrète évoquée par une sensation et des énoncés qui ont accompagné cette situation. Il peut également alors se concevoir avec une permanence (liée à la permanence de l'objet chez Piaget) et conserver une intégrité, une identité dans le temps : Comme l'écrit Ricœur dans Le soi comme un autre:

«Il ne s'agit pas de s'assurer qu'on parle de la même chose, mais qu'on peut l'identifier comme étant la même chose dans la multiplicité de ses occurrences. Or cela ne se fait que par repérage spacio-temporel : la chose reste la même en des lieux et des temps différents. (...) Même veut alors dire unique et récurrent. » (Ricœur 1990).

L'enfant fusionne ainsi les différents lui-même qu'il a été (et sera) dans le temps. Cela donne des énoncés dans lesquels l'enfant est en quelque sorte linguiste et dit "quand j'étais petit, je disais ta, maintenant je dis table". Mais il fusionne aussi les différentes représentations qu'il a et qu'on lui a données de lui-même.

L'ensemble de ce processus permet à l'enfant de s'approprier la parole de l'autre, notamment celle de sa mère, de souligner sa part d'écart par rapport à cet 
autre, et de faire entendre sa propre voix, construisant et marquant verbalement ainsi son identité propre.

Cette étude tentait donc d'analyser ces phénomènes d'écarts par rapport à l'auto-désignation employée dans la langue adulte que sont les emplois de l'accusatif et du génitif, les emplois de la deuxième personne et de la troisième personne. L'analyse de ces écarts nous amène à travailler sur la notion de sujet à la fois sujet grammatical et sujet parlant et sa mise en fonctionnement dans le langage de l'enfant.

A la fin du processus d'acquisition, vers 3 ans, l'enfant conjoint sujet de l'énoncé, sujet énonciateur, sujet sémantique, sujet de conversation dans une seule forme, je, mais il lui a fallu passer par un mouvement de disjonction de ces différents plans en produisant différentes marqueurs s'écartant de la forme adulte avant de les conjoindre.

Bibliographie

Bellugi, U. \& Klima, E. 1981. "From gesture to sign: Deixis in a visual-gestural language". In R.J. Jarvella and W. Klein (Eds.) Speech, place and action: Studies of languages in context. Chichester: John Wiley \& Sons.

Benveniste E., 1966, Problèmes de linguistique générale, volume 1, Paris, Gallimard.

Chiat, S. If I were you and you were me: the analysis of pronouns in a pronoun-reversing child. Journal of Child Language 9 1982. 359-379.

Chiat, S. Personal pronouns. In Language Acquisition, P. Fletcher et M. Garman (Eds). C.U.P. 1986.

Cuxac C., 2000, La langue des signes française, les voies de l'iconicité, Faits de Langues, $\mathrm{n}^{\circ} 15-16$, Paris, Ophrys.

Danon-Boileau, L. 1994. La personne comme indice de modalité. Faits de langues - la personne. Paris, P.U.F.

Danon-Boileau, L. 2002. Des enfants sans langage. Paris. Editions Odile Jacob.

Guillaume P. 1927. Les débuts de la phrase dans le langage de l'enfant, dans Journal de Psychologie Normale et Pathologique, no. 24, pp. 1-25, p. 1.

Morgenstern A., 1995, L'enfant apprenti-énonciateur, Thèse de doctorat nouveau régime dirigée par Laurent Danon-Boileau, Université Paris III.

Petitto L.A. 1984. From gesture to symbol: The relationship between form and meaning in the acquisition of personal pronouns in American Sign Language. Unpublished dissertation. Harvard University.

Ricœur, P. 1990. Soi-même comme un autre. Paris, Seuil.

Thom, R. 1980. Sur la typologie des langues naturelles: essai d'interprétation psycholinguistique, in Modèles mathématiques de la morphogenèse, pp.243-259, C. Bourgeois, Paris. 